\title{
Sweetest Protein -Thaumatin
}

\author{
NAWA RAJ DAHAL ${ }^{1,2 *}$ and $X . M . X^{1}$ \\ ${ }^{1}$ Southern Yangtze University, Wuxi City, Jiangsu, P. R. China \\ ${ }^{2}$ Department of Food Technology and Quality Control, Kathmandu, Nepal
}

Thaumatin, commercially available under the trade name Talin, is known as a sweetest compound (100,000 times sweeter than sucrose on molar basis) which can be used not only as a sweetener but also as a flavor enhancer. This sweetener is a protein found in plant Thaumatococcus daniellii. Biotechnology has also seen as alternative method of production of thaumatin. The plant source of this protein is limited and very expensive. Although the sweetener is the sweetest one, its application is not so common because of its liquorice taste and delayed sweetness. This article reviews briefly on the property, production, application and safety aspects of Thaumatin which is known as a sweetest compound till date.

Keywords: Thaumatin, Property, Production, Application, Safety aspect

\section{Introduction}

Sweeteners play a very important role as food additive. Nutritive sweeteners from the carbohydrate source are common from a long ago along with the invention of alternative sweeteners (Poldermans and Matt, 1991; Smith, 1991; Wel, 1993). Nonnutritive sweeteners have al.so known to play a crucial role as food additive especial.ly to those people who have prohibited consuming nutritive sweeteners. The other important point is that these nonnutritive sweeteners are very sweet as compared to the nutritive sweeteners and are relatively cheap (Higginbotham, 1979; Shllenberger, 1993; Gibbs et al., 1996) but the artificial one should pass the safety test and should be consumed only within the permitted limit Natural sources are safer than the artificial one. So the invention of natural, nonnutritive and intense sweeteners may occupy a feasible and more stable source of the sweeteners in the future. Sweetener known as Thaumatin has found such natural, non-nutritive and intense sweetener (Shallenberger, 1993; Daniell et al., 2000; Saumi et al., 1997).

Thaumatin is a mixture of intensely sweet tasting protein extracted from the fruit Thaumatococcus danicllii. (Poldermans and Matt, 1991; Smith, 1991) and it is available commercial.ly under the trade name Talin. (Wells 1989; Saumi et al., 1997) The major sweet tasting thaumatin proteins are thaumatin I and thaumatin II along with thaumatin a, thaumatin $b$ and thaumatin c. Thaumatin is by far the sweetest compound known till now (Poldermans and Matt, 1991; Smith, 1991; Wel, 2003). The aqueous solution of thaumatin range from 1300 times sweeter than sucrose when compared at a $13 \%$ concentration to about 5500 times sweeter when compared at $0.6 \%$ (Poldermans and Matt, 1991). It binds very strongly to the taste cell membrane. On molar basis thaumatin is 100,000 times sweeter than sucrose (Table 1). The threshold value is $10-8 \mathrm{~mol} /$ lit suggesting a strong affinity for receptor (Wel, 1993). Thaumatin has been reported to be synergism with other

** Corresponding author, E-mail: nawarajd@yahoo.com sweeteners such as saccharin (masks saccharin after taste), Akesulfate $\mathrm{K}$ and sterioside but it did not shown synergism with cyclamate or aspartame (Smith, 1991).

Table 1. Relative sweetness of Thaumatin

\begin{tabular}{lc}
\hline \multicolumn{1}{c}{ Sweetener } & Relative Sweetness \\
\hline Sucrose & 1.0 \\
Cyclamate & 50 \\
Saccharin & 300 \\
Thaumatin & 3,000 \\
& $(100,000$ times on molar basis $)$.
\end{tabular}

Source: Poldermans and Matt, 1991; Shallenberger, 1993

Thaumatin was firstly isolated by Van Der Wel in 1972 (Higginbotham, 1979; Smith, 1991; Gibbs et al., 1996). It has been marketed in Japan since 1979 and has been approved in the United States by the flavor extract manufacturers Association for use in chewing gum. In Canada, Thaumatins is permitted in animal feeds, chewing gum, flavor preparation, breadth sweetness, and sal.t substitute. In 1983, the use of thaumatin was approved by Great Britain for dietary products, drinks, pharmaceutical products and foods (excluding for babies) (Higginbotham, 1979; Shallenberger 1993; Gibs et al., 1996; Saumi et al., 1997). Now it is used as a natural intense sweeteners and flavors in food applications in many countries.

\section{Properties of Thaumatin}

Amino acid composition- The amino acid composition of Thaumatin shows that it is rich in glycine, aspartic acid, threonine, serine, alanine, cystine, arginini, phenylalanine (Table 2).

Solubility- Thaumatin is a cream colored powder which is extremely soluble in water enabling $60 \%$ solutions to be prepared (Higginbotham, 1979; Wells, 1989) but it is not soluble in organic solvent. 
Table 2. Amino acid composition (residues/mol) of thaumatin

\begin{tabular}{lcc}
\hline Amino acid & Thaumatin I & Thaumatin II \\
\hline Aspartic acid & 21 & 19 \\
Glutamic acid & 10 & 10 \\
Serine & 12 & 10 \\
Threonine & 19 & 17 \\
Proline & 12 & 12 \\
Glycine & 23 & 22 \\
Alanine & 15 & 14 \\
Valine & 9 & 8 \\
Leucine & 9 & 9 \\
Isoleucine & 7 & 7 \\
Methionine & 1 & 1 \\
Phenylalanine & 10 & 10 \\
Tyrosine & 7 & 8 \\
Half-cystine & 14 & 14 \\
Histidine & 0 & 0 \\
Lysine & 10 & 11 \\
Arginine & 11 & 12 \\
Tryptophan & 3 & 3 \\
Total & 193 & 187 \\
\hline Source Higginbotham & $(1979)$ &
\end{tabular}

Source: Higginbotham, (1979)
Sweetness characteristics- The sweetness time/intensity profile of thaumatin differs considerably from that of sucrose (Wells, 1989). There is a slow onset of sweetness which builds up to a maximum intensity followed by a long lingering sweetliquorice like aftertaste. It does not, however, have the bitter, metallic aftertaste associated with some of the other intense sweeteners (Wells 1989; Saumi et al., 1997). Cold aqueous solution of the mixed thaumatins have a sweetness ranging from 5500 times $0.6 \%$ sucrose at its threshold sweetness to 1300 times at $13 \%$ sucrose but separated TI and TII both slightly less sweet and TI less sweet then TII with $\mathrm{Ta}, \mathrm{Tb}$ and Tc also being even less sweet than TI, it would indicate that increasing basicity confers greater sweetness (Higginbotham, 1979; Linke 1987, Lindsay, 1996; Fisher and Scott, 1997; Coiffard et al., 1998). Sweetness property of thaumatin is given in Table 3 .

Sweetness stability- When subjected to boiling in deionised water, thaumatins show very little loss of sweetness even after several hours (Higginbotham, 1979; Shallenberger, 1993). Acetylation of only 4 of the 10 E-amino groups of lysine reduced sweetness zero. The property where by thaumatin I can be believed for more than an hour and then after cooling region its sweetness is proportional to the presence of its eight disulphide bonds (Gibbs et al., 1996; Saumi et al., 1997). Thaumatin is extremely stable in aqueous solution in the $\mathrm{pH}$ range 2.7-6.0 with an optimum around $\mathrm{pH}$ 2.8-3.0 which is ideal for soft drinks application. At low $\mathrm{pH}$ solutions, can be heated at $100^{\circ} \mathrm{C}$ for several hours without loss of sweetness (Wells, 1989; Schiffman et al., 2001).

Table 3. Sweeteness property of thaumatin

\begin{tabular}{lcc}
\hline \multicolumn{1}{c}{ Property } & Thaumatin I & Thaumatin II \\
\hline Sweetness intensity (times sweeter than sucrose) & & \\
On molar basis (a) at sweetness threshold (0.6\% sucrose) & $3 \times 10^{5}$ & $3.4 \times 10^{5}$ \\
(b) at 8\% equivalent sucrose concentration & $2 \times 10^{5}$ & $2.2 \times 10^{5}$ \\
On weight basis (a) at sweetness threshold (0.6\% sucrose) & 5000 & 5500 \\
(b) at 8\% equivalent sucrose concentration & 2500 & 2700 \\
Isoelectric point & 12 & 12 \\
Molecular weight & $21000 \pm 600$ & $20500 \pm 600$ \\
Absorption at 277 nm, at 0.1\%, pH 5, 1-cm cell & 1.27 & 1.22 \\
\hline
\end{tabular}

Source: Higginbotham, (1979)

Effect of salt- Both cations and anions are important with sodium sulphate being 3 times more effective than sodium chloride and calcium chloride (Higginbotham, 1979; Overbecke, 1989; Gibbs et al., 1996). Even though the effect is quite marked at comparatively low salt molarities $\left(10^{-2}\right.$ to 10 $\left.{ }^{3}\right)$ the relative molarity to thaumatin is large up to 3000 times being necessary to reduce sweetness (Table 5). The optimum sensitivity of thaumatin in aqueous solution was obtained at $277 \mathrm{~nm}$. A linear calibration (Correlation coefficient, $r>0.99$ ) was obtained over thaumatin concentration ranging from 4.8 $\times 10^{-6}$ to $3.8 \times 10^{-5} \mathrm{M}\left(=25,000 \mathrm{M}^{-1} \times \mathrm{cm}^{-1}\right)$ (Higginbotham, 1979).

Effect of temperature- The thermo-degradation of thaumatin in aqueous diluted solution for $\mathrm{pH}$ value was exposed on the rate of change of absorbance at $277 \mathrm{~nm}$. UV spectra obtained at $\mathrm{pH} 2$ which demonstrated the gradual decrease in absorbance at $277 \mathrm{~nm}$ during thermal treatment. The thermo-degradation 
Dahal \& Xu : J. Food Sci. \& Technol. Nepal, Vol. 7 (112-118), 2012

Table 4. Salt levels required to suppress taste of thaumatin

\begin{tabular}{lcccc}
\hline \multicolumn{1}{c}{ Salt } & Effective molarity & $\begin{array}{c}\text { Relative molarity } \\
\text { to thaumatin }\end{array}$ & $\begin{array}{c}\text { Cation concentration } \\
\text { ratio to thaumatin }\end{array}$ & $\begin{array}{c}\text { No. of ions per } \\
\text { amino acid }\end{array}$ \\
\hline $\mathrm{NaCl}$ & $1.9 \times 10^{-2}$ & $7800 \times$ & $3100 \times$ & 16 \\
$\mathrm{Na}_{2} \mathrm{SO}_{4}$ & $5.6 \times 10^{-3}$ & $2300 \times$ & $760 \times$ & 4 \\
$\mathrm{CH}_{3} \mathrm{COONa}$ & $1.8 \times 10^{-3}$ & $750 \times$ & $210 \times$ & 1 \\
$\mathrm{CaCl}_{2}$ & $4.6 \times 10^{-3}$ & $1800 \times$ & $670 \times$ & 3 \\
$\left(\mathrm{CH}_{3} \mathrm{COO}\right){ }_{2} \mathrm{Ca}$ & $1.6 \times 10^{-3}$ & $670 \times$ & $170 \times$ & 1 \\
\hline
\end{tabular}

Source: Higginbotham, (1979)

of thaumatin in aqueous diluted solution follow app. First order kinetics as described by the equation $\mathrm{C} / \mathrm{Co}=\mathrm{e}^{-\mathrm{kt}}$ where $\mathrm{C}$ and $\mathrm{Co}$ are the respective thaumatin concentration at time $\mathrm{t}$ and initial.ly, and $\mathrm{k}$ the app $1^{\text {st }}$ order degradation rate constant. Sweetness et al., 1 possible intensity level $(2.5 \%, 5 \%, 7.5 \%$ and $10 \%$ sucrose equivalent) was tested at warm $50^{\circ} \mathrm{C}$ and the cold $6^{\circ} \mathrm{C}$ temperature.

Effect of $\boldsymbol{p H}$ - Thaumatin is maximally stable at $\mathrm{pH} 2.0$ (Higginbotham, 1979).

Compatibility- The high overall positive ionic charge on the thaumatin molecule causes it to associate with suitably shaped, negatively charged ingredients such as synthetic colors and food gums (Higginbotham, 1979; Shallenberger, 1993; Wel, 1993). Synthetic colors having suitably spaced sulphonate groups can under certain condition of temperature, $\mathrm{pH}$ and concentration, link thaumatin molecule together eventual. ly causing precipitation. Even if precipitation doesn't occur, there may be a loss of color. The interaction of thaumatin with synthetic colors can be precipitated by the use of gum (Wells, 1989; Kuznetsor et al., 1995). Loss of sweetness can occur with gums such as xanthan, pectin, CMC, carrageenan etc when they are present in great excess. Thaumatin can enhance certain flavors, particularly peppermint spearmint, coffee and ginger flavor. It can al.so enhance aromas. Flavor enhancement can be achieved at levels below the sweet taste threshold (Wells, 1989; Shallenberger, 1993).

Caloric value- Since thaumatin is a protein, it does have an energy value of $4 \mathrm{Kcal} / \mathrm{gm}$. However because of its high sweetness potency, the caloric value per unit of sweetness is less than $0.002 \mathrm{kcal}$, so its contribution is negligible (Wells, 1989; Shallenberger, 1993).

Cariogenicity- Thaumatin is non-cariogenic but it is not cariostatic or anti-cariogenic (Wells, 1989).

Synergism- Thaumatin is synergistic with saccharin, acesulfame-K and stevioside but not with cyclamate or aspartame. It can be used to mask the bitterness of sweetness such as saccharine, as its sweetness is still perceived when the bitterness of saccharin would normal.ly be at a max (Higginbotham, 1979; Wells, 1989; Shallenberger, 1993).

\section{Production of Thaumatin}

There are two methods identified for production of Thaumatin. First method involves the isolation and purification from plant Thaumatococcus danielli and second method involves production from the biotechnological innovations.

Isolation and purification of Thaumatin from plant sourceVander Wel and Loeve in 1972 isolated two sweet tasting proteins named as Thaumatin I and II. The procedure adopted is given in Figure 1.

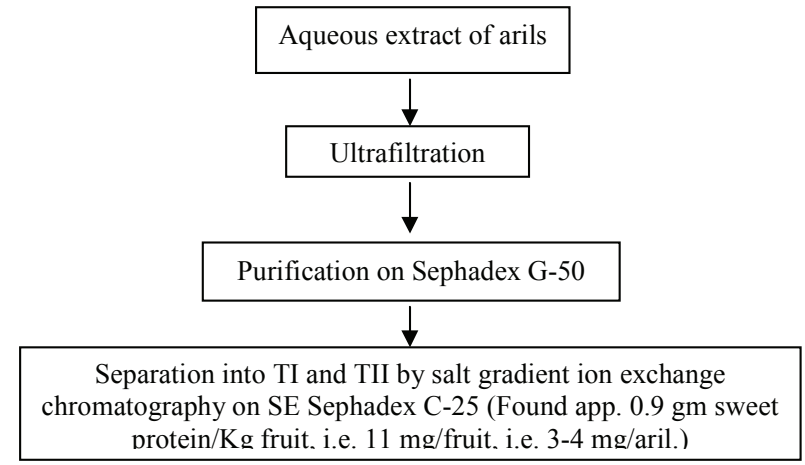

Figure 1. Isolation of plant Thaumatin

(Source: Higginbotham, 1979)

Thaumatin can accumulate up to $50 \%$ of the dry weight of aril in the fruit but not found in another part of the plant (Gibbs et $a l ., 1996)$. The study on the effect of salt and $\mathrm{pH}$ on yields was undertaken leading to a patient in 1977 on the production of Thaumatin. The study showed that yields couldn't be greatly increased by low concentration of salt in particular aluminum salts, which al.so improves the color, stability, filterability, purity and sweetness of the extracts. This had increased yields to $6 \mathrm{~g}$ sweet protein per Kg fruit (Higginbotham, 1979; Shallenberger, 1993; Gibbs et al., 1996).

If the thaumatin are carefully fractionated on what man CM 32 cation exchange in the $\mathrm{Na}+$ from using a shallow salt gradient at least 3 other distinct sweet proteins designed as $\mathrm{Ta}, \mathrm{Tb}$ and Tc can be distinguished eluted just before TI and TII. They all have similar in amino acid content and mol weight 18,000 to 20,000 but are less sweet than TI \& TII (Higginbotham, 1979). 
Production of Thaumatin from biotechnological innovationDespite the advantages of thaumatin protein in industrial use, thaumatin of plant origin is very limited because of the extreme difficulty involved in obtaining the fruit from which it is extracted (Faus et al., 1996). The producing plant $T$. daniellii, not only require a tropical climate and pollution by insects, but it must also be cultivated among other trees and yet 75\% of its flowers don't bear fruit (Faus et al., 1996; Gibbs et al., 1996). So to reduce heterogeneity in thaumatin preparation, ease purification procedures and to reduce the production time and cost of production, another approach of biotechnology for the production has been investigated that involves the transfer of its gene to another host such as bacteria like E. coli, Bacillus cereus and others (Gibbs et al., 1996; Saumi et al., 1997). The experiment has al.so been done to introduce gene into Saccharomyces cerevisiae to produce up to $0.15 \mathrm{~g} /$ lit of thaumatin intracellularly. Chemical denaturation and renaturation is necessary to obtain sweetness from the produced thaumatin (Overbecke, 1989; Kuznetsor et al., 1995; Gibbs et al., 1996).

Additional vectors such as an invertase signal sequence were utilized to secrete sweet thaumatin into the growth medium
$(300 \mathrm{mg} / \mathrm{L})$. The thaumatin produced in this manner was identical to plant thaumatin according to the chemical and taste test. The level of production approaches feasibility. In addition changes in the genetic sequence leads to variant with different taste properties (Gibbs et al., 1996; Kuznetsor et al., 1995). Microbial thaumatin has to be recovered probably after modification by enzymes, and then separated from cell components and other proteins and genetic material. (Higginbotham, 1983). A simple protocol for the biotechnology for the production of thaumatin is given by Weickmann et al., 1989 is given in Figure 2. The sequence of production is as follows.

- Coding sequence based on the amino acid sequence of thaumatin I was designed, synthesized and cloned into the appropriate vector (Figure 2). Yeast preferred codons were used so that the gene could be expressed efficiently in yeast. In order to express the thaumatin gene in yeast, a strong yeast promoter the 3-phosphoglycerate kinase (PGK) promoter was joined to the 5'end of thaumatin I, A and B genes. Plasmid containing the thaumatin $\mathrm{A}$ and $\mathrm{B}$ expression cassette were transferred into the yeast strain AH22 and BB25-1d.

- Cells were grown in sterile media under fermentation

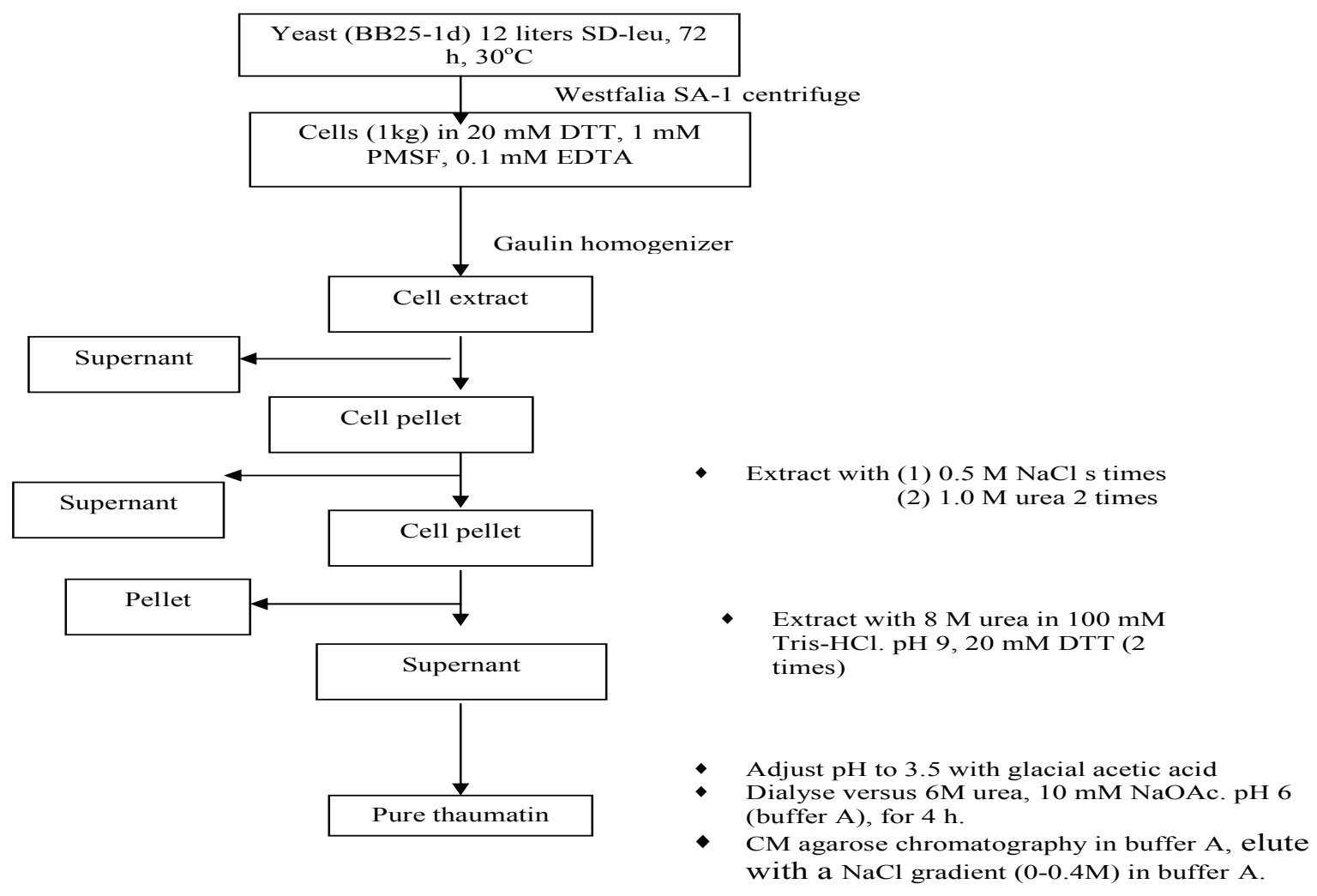

Figure 2. Protocol used for the isolation and purification of thaumatin from yeast cells (DTT= dithiothreitol,

Source: Weickmann et al., (1989) 
condition, harvested by centrifugation and lysed and the resulting extract was analyzed. The major protein recognized by the antiserum was located in the insoluble portion of the yeast extract, it co migrated with a plant thaumatin standard.

- Yeast transferred with the thaumatin expression vector produced about $20 \%$ of the insoluble protein (or $10 \%$ of the total. protein) as thaumatin. The insolubility and charge characteristics of thaumatin can be found advantageous during purification. The sequence of steps used to obtain $80 \%$ yields of $>95 \%$ pure thaumatin (Figure 2).

Improvement in the level of thaumatin secretion has allowed using fermentation and protein purification as a means of producing thaumatin and its derivatives on a large scale (Weickmann et al., 1989). A synthetic gene encoding the amino acid sequence of the strongly sweet tasting protein thaumatin II has been expressed in E. coli, The recombinant protein has been renatured from inclusion bodies using a reduced/oxidized glutathione system to yield a purified protein preparation that was indistinguishable from native thaumatin with respect to its biochemical spectroscopic and organoleptic properties (Daniell et al., 2000). The taste test of yeast thaumatin was determined that the sweetness threshold for plant thaumatin to be $2.3 \pm 0.9$ Microgram /lit. Similar results were obtained with thaumatin produced from yeast by either protein folding or secretion. One of the limitations of the plant thaumatin in a widely used sweetener is its persistent after taste. This property may prove to be advantageous for some applications like chewing gum, toothpaste etc., but for most products aftertaste is undesirable. A challenge to biotechnology is to design changes in the amino acid sequence of thaumatin to provide variants which retain the sweetness potency of the plant protein yet exhibit a reduced aftertaste (Weickmann et al., 1989). Specific changes were introduced into the thaumatin gene by mutagenesis in vitro by replacing DNA sequence by insertion of synthesized oligonucleotides (Weickmann et al., 1989; Wells 1989).

Although attempts have been made to produce thaumatins by genetic engineering in bacteria such as E. coli, Bacillus subtilis and Strepromyces lividans, in yeast such as Saccharomyces cerevisiae, and Kluveromyces lactis, in the fungus Aspergillus oryzae and in transgenic plants such as Solanum tuberosum, until now the results have been considered disheartening, thus the thaumatin available to industry is very scarce and expensive (Kuznetsor et al., 1995; Faus et al., 1996).

\section{Application of Thaumatin}

As sweeteners, thaumatin is used in beverages and desserts but its application are limited because of its liquorice taste and delayed sweetness. In practice therefore thaumatin is most commonly used as a practical sweetener mixed with other more rapidly tasting sweeteners (Higginbotham, 1979; Smith, 1991; Wel, 1993). Despite its limitations as sweeteners, thaumatin is a powerful flavor enhancer and manifest speraut cinnamon, wintergreen and peppermint by up to 10 times. This flavor potentially characteristics together with the lingering sweet taste can be beneficially used for products such as tooth paste, mouthwash and chewing gum and for enhancing the flavor medicines (Smith, 1991).

Thaumatin is used as a flavor enhancer in the following products.

- Japanese products- Firstly it was observed that Talin enhanced the flavor of peppermint; many practical applications have been developed. Flavor enhancement is noted when Talin is used at concentration below it sweet taste threshold-typically at $5 \times 10^{-5} \%$ Tal.in. Cost reduction of sugar and improved aroma are described for beverages, soft drinks, fruit juices, milk drinks, ice candies, sherbets, ice-creams, jellies, custard, chewing gum, wafers, soft candies, cream, pickles, fish paste, sauces, cheese and dried fish (Higginbotham, 1979; Gibbs et al., 1996).

- Chewing gum- Talin is used at 50-150 ppm the flavor of chewing gum- especially in the peppermint and spearmint types and the coffee and the fruit flavours such as lemon, plum and straw berry is enhanced and prolonged (Shallenberger, 1993; Gibbs et al., 1996).

- Coffee- The addition of very low level of Talin to instant coffee granules (made by re-drying strong coffee solution) enhances coffee strength by $10-15 \%$ (Higginbotham, 1979; Shallenberger, 1993).

- $\quad$ Soft drinks- The taste of Talin restricts its use in carbonated beverages to around 10-15 ppm, when it contributes up to $30 \%$ of the sweetness depending on the acidity and type of the drink. A reduction of sugar level in a syrup drink (normally over 145\% sucrose) not only reduces its calorie content and cariogenicity but al.so reduces, stickiness and improves flavor perception such levels also allow a reduction of $10-15 \%$ in the citric acid concentration used (Higginbotham, 1983).

- Flavor essences and oils- The solubility of Talin in aqueous alcohol allows its direct addition to the flavour essence or oil providing, a convenient route for its addition to chewing gum. Direct addition of Talin in 50\% aqueous alcohol to the flavour essences results in enhanced aroma quality and persistence (Higginbotham, 1983; Smith, 1991; Shallenberger, 1993).

- Medicines and oral care products- The intense sweet taste, non cariogenicity and flavour/aroma enhancement property of Talin suggest applications 
in improving the palatability of medicines particularly those with unpleasant-tasting long lasting ingredients not easily masked by sugar or glucose syrups alone. Level of use can start as low as $0.005 \%$ in liquid preparations where flavour enhancement and taste masking are the main requirement and can rise to $0.05 \%$ or even higher when particularly strong tastes are present and strong sweetness is required (Higginbotham, 1983; Smith 1991; Shallenberger, 1993)

\section{Safety aspects}

The safety of thaumatin has been proven for animal and humans. It doesn't cause tooth decay and can be used by diabetes (Higginbotham, 1983; Gibbs et al., 1996). Thaumatin is the only natural high intensity sweeteners and product containing it does not require to be labeled as artificial sweeteners. It has a low calorie value and is non-cariogenic. FAO/WHO expert committee recorded no mutagenic, terratogenic or allergenic effect of thaumatin and concluded that the lack of toxicity together with its ready digestion to normal food component indicated that its only dietary effect was to make an insignificant contribution to the normal protein intake (Smith, 1991). The toxicological studies indicate a safety factor of at least 30,000 times the expected daily human intake level (Higginbotham, 1983)

\section{Concluding remarks}

Thaumatin was first isolated from the plant Thaumatococcus daniellii by Van der wel and Loeve and its unique property of sweetness has been identified as being the sweetest compound in the world till to date The extensive research then had been carried out. It has realized on the basis of its research outcome is that the plant source of the protein is very expensive and al.so the taste property of this protein was slightly inferior as compared to sucrose. A strategy to use as a mixture to other sweeteners was also done and some sweetener like saccharin, acesulfame-K and stevioside (but not with cyclamate or aspartame) showed as a synergism effect to thaumatin. An important fact about thaumatin is that it can also be used as a good flavor enhancer. These two properties (sweetener and flavor enhancer) have also given the more importance to this additive to be used in food products. This condition leads the scientific community to look after the alternative source of the plant thaumatin and also to improve the taste and flavor property of this sweetener. Biotechnology has seemed a good alternative tool. Although the various works had been carried out, the outcomes are still on progress. Further improvement in biotechnological investigations may lead to new era on the effective application of the thaumatin to be used both as a unique sweetener and a flavor enhancer in the near future.

\section{References}

Coiffard C. A., Coiffard L. J. and Rock- Holt Zauer D. R. (1998). Influence of $\mathrm{pH}$ on thermo-degradation of thaumatin in aqueous solution. Food Research International, 30: 707-710.

Daniell S., Mellits K. H, Faus I, Connerton I. (2000). Refolding the sweet protein Thaumatin II from insoluble inclusion bodies synthesized in E. coli. Food Chem., 71: 105-110.

Faus I., Patino C., Rio J. C. D., Moral. C. D., Barroso H. S. and Rubio V. (1996). Expression of a synthetic gene encoding the sweet tasting protein Thaumatin in E. coli. Biochemical and Biophysical Research Communication, 229:121-127.

Fisher C. and Scott T. R. (1997). Food flavors. Biology and Chemistry. The Royal Society of Chemists, pp. 128.

Gibbs B. F., Alli I. and Mulligan C. (1996). Sweet and Taste Modifying Proteins- A review. Nutrition Res., 16: 16191630.

Higginbotham J. D. (1979). Protein Sweeteners. In: Developments in sweeteners-1, Ed by Hough C. A. M, Parker K. J. and Vlitos A. J., Applied Science Publishers Ltd. London pp 87-123.

Higginbotham J. D. (1983). Recent developments in nonnutritive sweeteners. In: Developments in Sweeteners-2. Ed. by. Grenby T.H., Parker K.J. and Undley M.G. Applied Science Publishers, pp. 119-155.

Kuznetsor Y. G., Mal.kin A. J., Greenwood A. and Mc Pherson A. (1995). Interferometric studies of growth kinetics and surface morphology in Macromolecular Crystal growth, canaval.in, Thaumatin and Turnip Yellow Mosaic Virus. J. of Structural. Bio., 14:184-196.

Lindsay R.C. (1996). Food Additives. In: Food Chemistry, $3^{\text {rd }}$ Edition, Ed., Fennema O. R. Marcel Dekker Inc., pp. 798.

Linke H. A. B. (1987). Sweeteness and Dental health the influence of sugar substitutes on oral microorganisms. In: Developments in sweeteners-3. Ed. By Grenby T.N. Elsevier Applied Sci. pp 179-182.

Overbecke N. (1989). Genetic Engineering in flavour research. In: Biotechnology Challenges for the flavor and food industry. Ed. By Lindsay R. C. \& Willis B. J., Elsevier Applied Sci., pp.91-92.

Poldermans B., Matt J. (1991). Processing aids for the food industry: The state of the art, In: Boitechnological innovations in Food processings. Open universitiet and Thames Polytechnique, pp. 7-44.

Saumi T., Hough L., Machinami T., Watanabe N. \& Nakamura 
R. (1997). Molecular mechanism of sweet taste 7: The sweet protein, Thaumatin I. Food Chem., 60: 277-285.

Schiffman S. S., Sattely- Miller E. A., Graham B. G., Bennet J. C., Booth B. J., Desai N. and Bishay I. (2001). Effect of temperature, $\mathrm{pH}$ and ions on sweet taste. Physiology and Behaviour, 68:469-481.

Shallenberger R. S. (1993). Taste Chemistry. Blackie Academic and Professional, pp. 251-252.

Smith J. (1991). Food Additive User's Handbook. Blackie and Sons, pp. 68-69.

Weickmann J. L., Lee J. H., Blair L. C., Dasti Dar P. G. and
Koduri R. K. (1989). Exploitation of genetic engineering to produce novel protein sweeteners. In: Progress in sweeteners, Ed. By. Grenby T. H., Elsevier Applied Science, pp. 47-69.

Wel H. V. D. (1993). Some thoughts about Thaumatin Binding. In Sweet taste Chemoreception. Edited by Mathlouthi M, Kanters J. A. and Birch G. G. Elsevier Applied Science pp. 365-372.

Wells A. G. (1989). The use of intense sweeteners in soft drinks. In: Progress in sweeteners. Ed by Grenby T. H., Elsevier Applied Sci., pp. 185-186. 